\section{Organelle transport}

\section{Retrograde step for microtubules}

\author{
Jeremy S. Hyams
}

LOOKING at a living cell through the microscope is a bit like having a bird's eye view of Piccadilly Circus in rush hour. Vesicles and organelles show a complex two-way traffic pattern along intracellular highways of microtubules radiating out from the nucleus. Movement is either outwards (anterograde), towards the suburbs at the cell periphery, or inwards (retrograde), towards the bright lights at the cell centre. Once the black box of cell motility, organelle transport is rapidly becoming its star performer, at least as judged by the frequency of News and Views articles devoted to it. What has brought about this dramatic turn-around has been the ability to reconstitute particle movements in vitro ${ }^{1.2}$. The first significant success of this approach was the identification of a protein, kinesin, which has at least some of the properties expected of the anterograde organelle translocator ${ }^{3}$. Now, in the September issue of the Jour nal of Cell Biology $y^{4}$ and on page 181 of this issue ${ }^{5}$, Richard Vallee and colleagues report the isolation of a protein with impressive credentials for being the retrograde organelle motor.

What is totally unexpected about their discovery is that this latest microtubule motor activity is not associated with a 'new' protein. Instead it is a hitherto unsuspected property of MAP 1C, one of the five high-molecular-mass microtubule-associated proteins or MAPs previously described by that laboratory ${ }^{6}$. These proteins (the others are MAP 1A, MAP 1B, MAP 2A and MAP 2B) were originally identified in brain. Their abundance varies markedly between different regions of the brain and from one tissue type to another, although the significance of this is still not fully understood. MAP 1C in particular has remained enigmatic because of its low abundance under all preparative conditions.

What Vallee and his co-workers now show $^{4.5}$ is that, like kinesin, MAP $1 \mathrm{C}$ is greatly enriched in microtubules prepared in the absence of nucleotides. After selective removal of kinesin from their microtubule preparations using GTP, the authors could obtain a preparation highly enriched in MAP 1C by subsequent extraction of the microtubules with ATP. A microtubule-activated ATPase activity copurifies with MAP 1C, a clear indication that this protein might have a mechanochemical function ${ }^{4}$. To confirm this prediction, the authors turned to the remarkable ability of the AVEC microscope $^{7}$ to see the movement of single microtubules. When microtubules are placed on a glass microscope slide coated with MAP $1 \mathrm{C}$, microtubule gliding occurs in a continuous, unidirectional fashion ${ }^{4}$. The rate of about $1.25 \mu \mathrm{m} \mathrm{s}^{-1}$ is comparable to that recorded for organelle motility in living cells, but about four- to fivefold higher than previously reported for kinesin using the same assay ${ }^{8}$.

Most significantly, however, MAP 1C and kinesin generate force in opposite directions ${ }^{5}$. The touchstone of microtubule-polarity experiments are the axonemes of the green alga Chlamy domonas in which the plus and minus microtubule ends can be unambiguously distinguished (see Fig. 3 of ref. 5, on page 183). On MAP 1C-coated slides, Vallee and collaborators found axoneme gliding is always from the compact (proximal or minus) to the frayed (distal or plus) end. Kinesin, on the other hand, causes axonemes to glide in the opposite direction. Because in living cells microtubules are oriented with their plus ends distal to the organizing centre at the nucleus, force production by MAP $1 \mathrm{C}$ is in the retrograde direction. Thus, MAP $1 \mathrm{C}$ could be the molecular motor for endocytosis, for fast retrograde transport in neurons and even for the positioning of organelles such as the Golgi apparatus and lysosomes near

\section{Immunology}

\section{Jonathan Rothbard}

THE concentration of effort to develop safe, cheap and effective vaccines to viral, bacterial and parasitic pathogens refocuses attention on several well-established tenets of immunology: the generation of the humoral response requires cooperation between B and T lymphocytes ${ }^{1}$ (see figure); small-sized antigens induce immunity only when covalently attached to proteins that can be recognized by $T$ cells ${ }^{2.3}$; and immunological responsiveness is dependent on the HLA genotype of an individual ${ }^{4}$, which has now been correlated with the ability of an individual's histocompatibility (MHC) antigens to bind fragments of the immunogen ${ }^{5}$. Several recent papers describe the application of these principles to improve the use of synthetic peptides as vaccine components $^{6-9}$, the latest of which is reported on page 168 of this issue ${ }^{9}$ by Francis et al. of Wellcome laboratories. Whether these strategies will generate viable peptide vaccines remains to be seen, but the the nucleus of cells.

Exciting as these findings are, they may be only the start of a much more profound insight into the mechanisms of intracellular transport and, indeed, of other forms of cell motility. Ever since the isolation of flagellar dynein almost 25 years ago and the demonstration of its role in force generation in ciliary and flagellar movement, there has been an intensive search for 'cytoplasmic dyneins', high-molecularmass, microtubule-associated ATPases that might be expected to underlie the movement not only of organelles but also of chromosomes at mitosis and meiosis. MAP 1C may be just such a protein. Certainly, in terms of molecular mass, sedimentation coefficient, sensitivity to inhibitors and the finding that it can be specifically fragmented by ultraviolet light in the presence of vanadate, MAP $1 \mathrm{C}$ closely resembles flagellar dynein. Doubtless, experiments aimed at further establishing this relationship are already well in hand. Cell motility is once again a fast-moving field!

Brady, T., Lasek, R.J. \& Allen, R.D. Science 218 1129-1131 (1982)

2. Vale, R.D., Schnapp, B.J., Reese, T.S. \& Sheetz, M.P. Cell 40, 559-569(1985).

. Vale, R.D. et al. Cell 43, 623-632 (1985).

4. Paschal, B.M., Shpetner, H.S. \& Vallee, R.B. J. Cell Biol. $105,1273-1282$ (1987)

. Paschal, B.M. \& Vallee, R.B. Nature 330, 181 - 183 (1987)

6. Bloom, G.S., Schoenfeld, T.A. \& Vallee, R.B. J. Cell Biol 98, 320-330 (1984)

7. Allen, R.D. et al. J. Cell Biol. 100, 1736-1752 (1985).

Vale, R.D., Rees, T.S. \& Sheetz, M.P. Cell 42, 39-50 (1985)

Jeremy S. Hyams is Reader in Cell Biology in the Department of Biology, University College, London WC1E 6BT, UK.

\title{
Synthetic peptides as vaccines
}

experiments undoubtedly will generate useful research applications.

The groups attempting to develop vaccines against malaria ${ }^{6}$ and against footand-mouth virus ${ }^{9}$ have independently recognized that the linear peptides corresponding to protective antibody determinants are insufficient to immunize mice of divergent histocompatibility types. In either disease system, most strains of mice are non-responsive to the short linear peptides, but this problem can be overcome by covalently linking the peptide to a T-cell determinant capable of being recognized by the particular genetic strain of mouse.

In the case of malaria, Good and colleagues ${ }^{6}$ used a determinant within the circumsporozoite protein, whereas the Wellcome group 9 conjugated a defined helper determinant from a heterologous protein to improve the immunogenicity of their foot-and-mouth peptide. Although these experiments are natural sequelae 